\title{
A wireless sensor network location approach based on the characteristics of electromagnetic wave symmetry
}

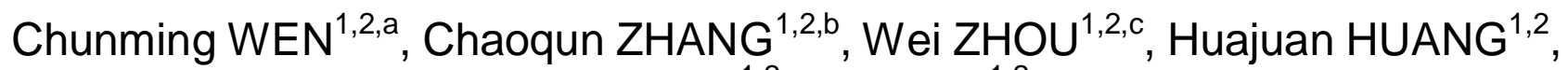 \\ Jing YANG ${ }^{1,2}$, Xiao QIN ${ }^{1,2}$
}

1.College of Information Science and Engineering, Guangxi University for Nationalities, Guangxi

Nanning 530006, China ;

\begin{abstract}
2.Guangxi Higher School Key Laboratory of Complex Systems and Intelligent Computing , Guangxi Nanning 530006, China
\end{abstract}

a wenchunming@gxun.edu.cn, b zhangchaoquni@gxun.edu.cnl, c zhouwei@gxun.edu.cnl

\begin{abstract}
Keywords: Wireless Sensor Network WSN, Location method, Electromagnetic wave, symmetrical propagation, RSSI

Abstract: Positioning plays an important role in wireless sensor networks. As a low-cost and low-power location approach, the RSSI (Received Signal Strength Indication) algorithm is widely used in wireless sensor networks location. However, the RSSI value is usually affected by environment conditions, easily leading to a large error. To eliminate the influence of environmental factors, and to improve positioning accuracy, this paper uses the symmetrical characteristics in electromagnetic wave propagation to design a location method to calculate the position of the node. The proposed method is tested with simulation experiments in MATLAB and the test results show that the new location method has a high positioning accuracy.
\end{abstract}

\section{Introduction}

Wireless sensor networks have broad applications in military reconnaissance, transport management, environmental monitoring, health care, industrial and agricultural production, and so on. Most applications involve geographical parameters. Without a good position parameter, its value is greatly depreciated, and sometimes even becomes meaningless. Therefore, location is very essential for wireless sensor networks.

Node localization in wireless sensor network is a process to determine the locations of other nodes in the network from the locations of a small number of known nodes with a certain technology. Many schemes have been proposed for node localization ${ }^{[1-2]}$. For example, RSSI-based location is a low-power, low-cost and convenient location method. It uses electromagnetic signal strength to measure distance and can be used to locate wireless sensor network. However, the precision of this method is relatively low and the stability is very poor.

Many improvements of RSSI location technology have been studied in recent years. Weizheng Ren et $\mathrm{al}^{[3]}$ use beacon node which is nearest to the target node as the reference node to differentially correct the ranging based on RSSI to improve the positioning accuracy. Yan Xu et al ${ }^{[4]}$ use solving nonlinear equations approach for multi-point RSSI value to optimize the location accuracy. Dengcai $\mathrm{Xu}^{[5]}$ improve positioning accuracy by the least squares method. jie Zhan ${ }^{[6]}$ use Gaussian fitting process to improve the positioning accuracy. Chengpei Tang et $\mathrm{al}^{[7]}$ improve positioning precision by the ways of overlapping positioning method. Senlin Cheng et $\mathrm{al}^{[8]}$ improve positioning accuracy with the probability centroid method. Yan $\mathrm{Shi}^{[9]}$ improve location performance by the method of signal strength matrix. Xijian $\mathrm{Chen}^{[10]}$, Yan $\mathrm{Xu}^{[11]}$, Yan $\mathrm{Zhou}^{[12]}$ et al also use different methods to improve the RSSI algorithm. Velimirovic Andrija $S^{[13]}$ enhance the positioning accuracy by fuzzy method to cope the uncertainty of RSSI value. Lasla $\mathrm{N}^{[14]}$ indicates the position of the test nodes with geometry set based semi-symmetrical lens collection. Lim Jaechan ${ }^{[15]}$ improve positioning performance with a maximum likelihood (ML) estimation method. Bekcibasi Ugur et $\mathrm{al}^{[16]}$ improve positioning accuracy by increasing the reference anchor node method. Blumrosen $\mathrm{G}^{[17]}$ improve the positioning accuracy by using a calibration scheme ways. Schemes 
mentioned above are very helpful to improve the accuracy, but the improvement is very limited because the influence of environment changes and other interference factors is not considered yet.

Since node location is very important for wireless sensor, this paper proposes a positioning method based on the symmetry characteristics of electromagnetic wave propagation to solve the problems of poor stability and low accuracy in the RSSI algorithm which are caused by environmental factors. Then we process signals, and propose a strategy to select the matching anchor node and calculate the location information using electromagnetic wave propagation symmetry principles. The new method can eliminate the adverse effects of electromagnetic signal intensity changes in the RSSI location. It is low-power and low-cost and has a good stability.

\section{RSSI location principle}

The RSSI location method calculates the node position with trilateral location method. It applies the RSSI value to estimate the distance between adjacent points by electromagnetic propagation theory. During the transmission of electromagnetic signals, signal strength will gradually decay with the increase of transmission distance. In the RSSI ranging scheme, the transmitting power of the sending node is known and the received power is measured at the receiving node. Then the transmission loss can be calculated and converted to distance based on theoretical or empirical models. A useful formula is

$$
P L(d)=P L\left(d_{0}\right)-10 \mathrm{n} \lg \left(d / d_{0}\right)-X \sigma
$$

Where $d$ is the distance between the receiver and the sender, $d_{0}$ is a reference distance, $\mathrm{n}$ is the channel attenuation index, $X$ and $\sigma$ are the mean zero and variance of the Gaussian random noise, $P L(d)$ is the signal strength at d-distance from the sender, $P L\left(d_{0}\right)$ can be acquired from experience. The value of distance $d$ is obtained by substituting the values of signal intensity $P L(d)$ into (1) and solving the equation. Since the sensor nodes have the capability of wireless communication, and the RSSI data is acquired from the data packet, thus this location method does not require any additional hardware. Therefore, the RSSI location method is a convenient and inexpensive ranging technology with low power consumption.

However, atmosphere is not a lossless medium, molecules in the atmosphere, especially water vapor and oxygen, having their inherent electromagnetic harmonic vibration frequency, so the electromagnetic wave will have a high loss occurred in the harmonic frequency, thus the loss is related to the environmental factors such as temperature, humidity and altitude. In the real environment, due to the uncertainty of the external environment, the Gaussian random error X $\sigma$ values vary widely, resulting in a large positioning error,so the RSSI signal strength is very unstable and is easily influenced by environmental factors (e.g., temperature, humidity, etc.), this method may have a big ranging error.

\section{New positioning method}

Positioning error results from changing signal strength caused by environmental factors when the electromagnetic wave spread in the space. But the spread is symmetric and therefore the property can be used to offset the impact of signal intensity change. This paper groups several anchor nodes together through signal strength received by the test nodes. Anchor nodes with the same signal strength are put into one group. According to the symmetry characteristics of electromagnetic wave propagation along each direction in space, our new location method assumes that when the test node is equidistant from two anchor nodes, the strength of electromagnetic waves received from the two anchor nodes are also equal, even if the electromagnetic wave may be influenced by factors such as weather. Therefore, the position of the test node can be calculated by using the position parameters of the two anchor nodes. Figure 1 and 2 illustrate the principle.

Because the strength of electromagnetic waves that a test node receives from a tuple of anchor nodes in a certain group is equal, it can be assumed that the distance from the test node to the two anchor nodes are equal. Therefore, the test node $\mathrm{M}$ is located on a middle line which situates between the two anchor nodes (e.g., [A, B] or [C, D] in Figure 1) and is equidistant from the two 
anchor nodes. In other words, the test node $\mathrm{M}$ is located on the perpendicular bisector of the tuple. If the test node $M$ receives signal from multiple anchor node in the same group, then there will be a plurality of straight lines intersected with each other. Ideally, a plurality of straight lines intersect at only one point, and this point is exactly the position of the test node M. In practice, a plurality of straight lines may intersect at a plurality of points due to various factors and it may have more than one location. In this case one can determine the location parameter of the test nodes by Weighted Centroid Algorithm (the greater the signal strength value is, the heavier the weight is).

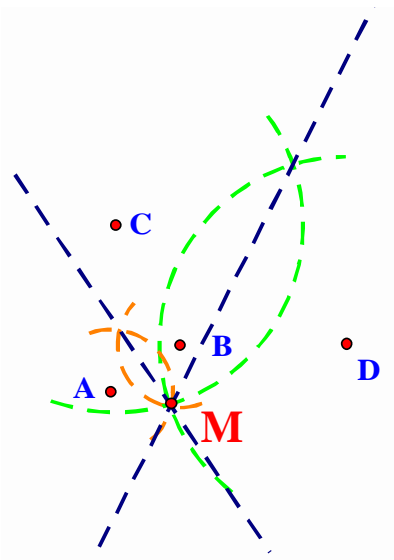

Fig. 1 Tested nodes $M$ is on the same side of anchor nodes A, B, C and D

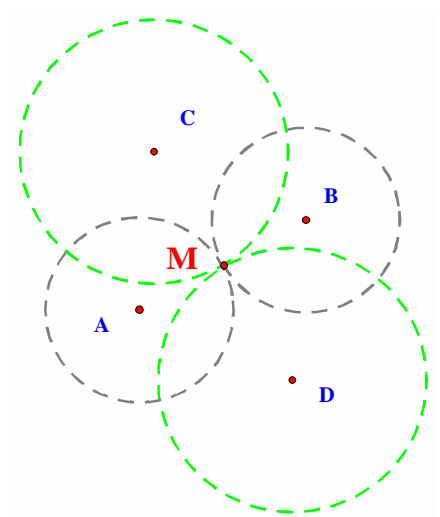

Fig. 2 Test node $M$ is in the middle of anchor nodes A, B, C and D

When the test node $\mathrm{M}$ is located at the center of anchor nodes, the center is the position of the test node. In this case, this method only involves a simple calculation and has a higher positioning accuracy. For positioning in the three-dimensional space, the test node will be located at perpendicular bisecting plane of symmetrical anchor nodes. The intersection of three symmetrical anchor nodes not in the same plane is exactly the position of the test node.

\section{Simulation}

The designed location scheme is implemented in MATLAB to simulate the new location solutions. The location range $(\mathrm{X} \times \mathrm{Y})$ is $1000 \mathrm{~m} \times 1000 \mathrm{~m}$. A number of test nodes and anchor nodes are randomly distributed in the region. Fig. 3 shows the comparison of the new symmetrical location method with the traditional RSSI trilateral location method.

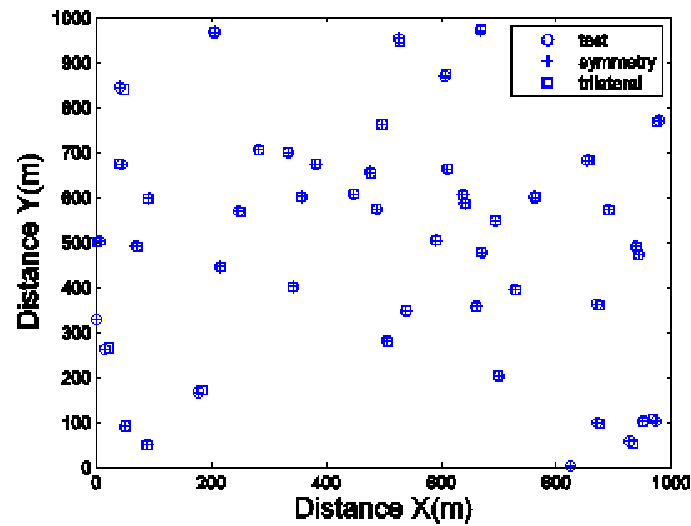

Fig. 3 Comparisons of symmetrical and trilateral location methods

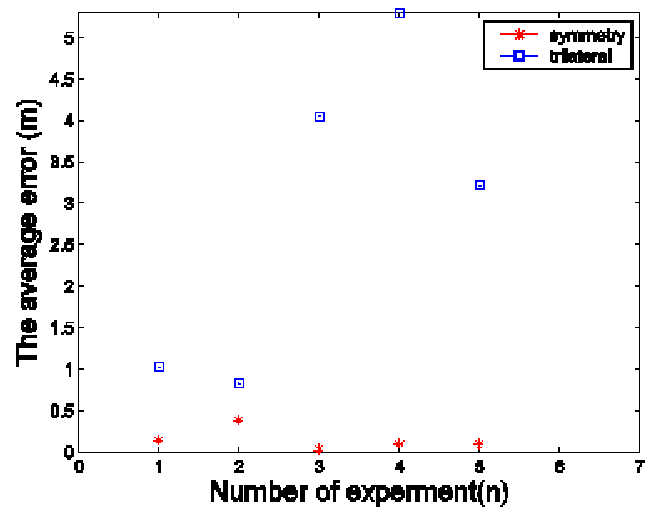

Fig. 4 Comparison of measurement errors between symmetrical and trilateral location methods.

In Fig.3, o represents the pre-set position of the test node, $\square$ is the test node's position obtained by the trilateration method and + is the test node's position obtained by symmetric method. The points measured by these two positioning methods are located nearby the random test point, but 
the points obtained by the symmetric approach are closer to the random test points, and its positioning error is smaller.

In Fig.4, *represents the positioning error of the symmetrical location method, $B$ is the positioning error of the trilateral location method. It shows the comparison of repetitive errors measured by using the two methods. As can be seen from the figure, the positioning accuracy of the proposed method is superior to the trilateral location method. The precision error is only $1 / 4$ of the traditional RSSI method. And its error value is only a little change, more stable. Thus it has a high positioning accuracy and better stability.

For the simulation range we use (i.e., $1000 \mathrm{~m}$ ), the maximum error is only a few meters.

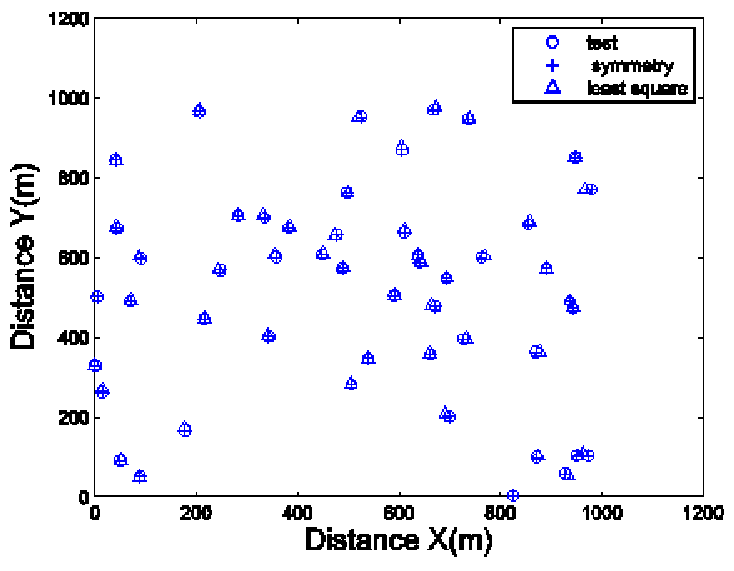

Fig.5 Comparisons of symmetrical and least-squares location methods.

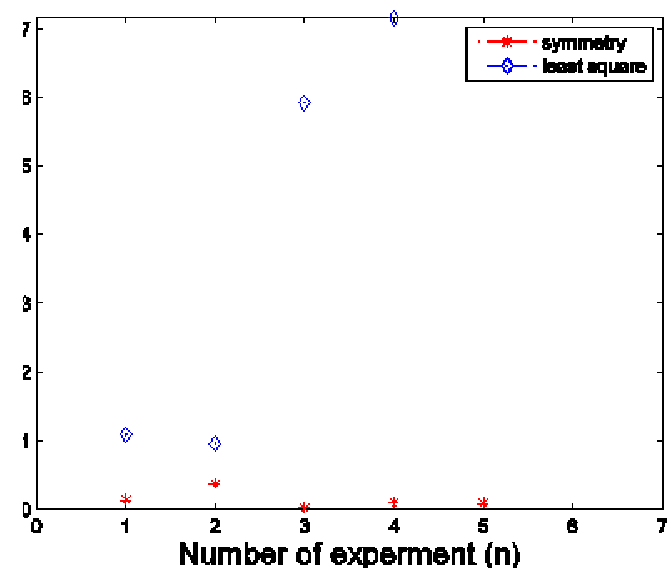

Fig.6 Comparison of measurement errors between symmetrical and least-squares location method.

Fig. 5 shows the comparison of the new symmetrical location method with the least-squares location method. In Fig.5, o represents the pre-set position of the test node, $\Delta$ is the test node's position obtained by the least-squares location method and + is the test node's position obtained by symmetric method. The points measured by these two positioning methods are located nearby the random test point, but the points obtained by the least squares method are a little far away from the random test points, and thus the positioning error to be slightly bigger.

In Fig.6, * represents the positioning error of the symmetrical location method, $\pi$ is the positioning error of the least squares location method. It shows the comparison of repetitive errors measured by using the two methods. As can be seen from the figure, the positioning accuracy of the proposed method is superior to the least-square. In repeated randomized trials, the errors are much smaller than the errors from the least square method.

\section{In conclusion}

This paper makes use of symmetry properties in the electromagnetic wave propagation along different directions in the space and proposes a new location method by using symmetrical anchor node. The new approach can eliminate the influence of environmental factors on the location, such as weather. Simulation results show that the proposed method can effectively improve the positioning accuracy and the calculation is very small. Furthermore, it does not require additional hardware devices while provide huge advantage. In particular, it can achieve high positioning accuracy when the test areas have a high anchor node density.

\section{Acknowledgements}

This work was supported by Science Foundation of Higher Education of Guangxi Zhuang Autonomous Region ( 2014YB102 ) ,Guangxi Higher School Key Laboratory of Complex Systems and Intelligent Computing(15CI08Y). 


\section{References}

[1] Sun L M, Li J Z, Chen Y, et al. Wireless Sensor Networks. Beijing: Tsinghua University Press, 2005.

[2] Kala S M, Reddy M P K, Musham R, et al. Interference mitigation in wireless mesh networks through radio co-location aware conflict graphs[J]. Wireless Networks, 2015, 32(3):1-24.

[3] Ren W Z, Xu L M, Deng Z L, et al. Distance Difference Localization Algorithm Based on RSSI for Wireless Sensor Networks. Chinese Journal of Sensors and Actuators. 2008, 21: 1247-1250.

[4] Xu Y, Shi J H, Wu X F. An Improved Localization Algorithm Based on RSSI-margin in WSN. Journal of Xiamen University(Natural Science), 2008, 47: 361-364.

[5] Xu D C, Zhou Q N, Zhang Z H. Research of RSSI location algorithm based on improved. Industrial Control Computer, 2013, 26: 72-73.

[6] Zhan J, Wireless sensor network technology to locate and track and Security Policy Studies. Changsha: Hunan University, 2011.

[7] Tang C P, Zhang H F, Yin J, et al. An RSSI-based wireless sensor network environment adaptive ring overlapping localization algorithm. China, CN103024897A, 2013-4-3.

[8] Chen S L, Li L Zhu B W, et al. Computing method of RSSI probability centroid for location in WSN. Journal of Zhejian University(engineering science ), 2014, 48: 100-104.

[9] Shi X, Yin A M, Chen X. RSSI and multidimensional scaling based indoor localization algorithm. Chinese Journal of Scientific Instrument, 2014, 35: 261-268.

[10] Chen X J, Cheng L L. Study and Implementation of Power Match Algorithm Based on RSSI. Chinese Journal of Sensors and Actuators, 2013, 26: 709-714.

[11] Xu Y, Shi J H, Wu X F. An Improved Localization Algorithm Based on RSSI-margin in WSN. Journal of Xiamen University(Natural Science. 2008, 47: 361-364. 3.

[12] Zhou Y, Li H C. Space localization algorithm based RSSI in wireless sensor networks. Journal on Communications, 2009, 30: 75-79.

[13] Velimirovic A S, Djordjevic G L, Velimirovic M M, et al. Fuzzy ring-overlapping range-free (FRORF) localization method for wireless sensor networks. Computer Communications, 2012, 35 : 1590-1600.

[14] Lasla N, Derhab A, Ouadjaout A, et al. Half-Symmetric Lens based localization algorithm for wireless sensor networks. 2012, 37th Annual IEEE Conference on Local Computer Networks (LCN 2012). 320-323.

[15] Lim J, Lee J. Asymptotic optimal method for localisation of a target in wireless sensor networks. International Journal of Communication Networks and Distributed Systems, 2009, 3: 36-54.

[16] Bekcibasi U, Tenruh M. Increasing RSSI Localization Accuracy with Distance Reference Anchor in Wireless Sensor Networks. Acta Polytechnica Hungarica, 2014, 11: 103-120.

[17] Blumrosen G, Hod B, Anker T, et al. Enhanced calibration technique for rssi-based ranging in body area networks. Ad Hoc Networks, 2013, 11: 555-569. 